\title{
484.
}

\section{ON THE VARIATIONS OF THE POSITION OF THE ORBIT IN THE PLANETARY THEORY.}

[From the Monthly Notices of the Royal Astronomical Society, vol. xxxII. (1871-72), pp. 206-211.]

IT has always appeared to me that in the Planetary Theory, more especially when the method of the variation of the elements is made use of, there is a difficulty as to the proper mode of dealing with the inclinations and longitudes of the nodes, hindering the ulterior development of the theory. Considering the case of two planets $m, m^{\prime}$, and referring their orbits to any fixed plane and fixed origin of longitudes therein, let $\theta, \theta^{\prime}$ be the longitudes of the nodes, $\phi, \phi^{\prime}$ the inclinations $(p=\tan \phi \sin \theta$, $q=\tan \phi \cos \theta$, \&c., as usual); then the disturbing functions for $m, m^{\prime}$ respectively are developed, not explicitly in terms of $\phi, \phi^{\prime}, \theta, \theta^{\prime}$, but in terms of $\Phi$, the mutual inclination of the two orbits, and of $\Theta, \Theta^{\prime}$ the longitudes in the two orbits respectively of the mutual node of the two orbits; $\Phi$ and $\Theta, \Theta^{\prime}$ being functions (and complicated ones) of $\phi, \phi^{\prime}, \theta, \theta^{\prime}$. Moreover, although in the general theory of the secular variations of the orbits of the planetary system, $\theta, \phi$, \&c., are, as above, referred to one fixed plane (the ecliptic of a certain date), yet in the theory of each particular planet it is the practice, and obviously the convenient one, to refer for such planet the $\theta, \phi$ to its own fixed plane (the orbit of the planet at a certain date), the effect of course being that $\phi$, and consequently $p$, $q$, instead of being of the order of the inclinations to the ecliptic, are only of the order of the disturbing forces. It has occurred to me that the last-mentioned plan should be adhered to throughout; viz., that for each planet $m$, the position of its variable orbit should be determined by $\theta$, the longitude of its node, and $\phi$, the inclination in reference to the appropriate fixed plane (orbit of the planet at a certain date) and origin of longitude therein. The disturbing functions for the planets $m$ and $m^{\prime}$ will of course depend not only on $\theta, \theta^{\prime}, \phi, \phi^{\prime}$, but on the quantities $\Phi, \Theta, \Theta^{\prime}$ which determine the mutual positions of the two fixed 
planes of reference and origins of longitude therein, these last being however absolute constants not affected by any variation of the elements; so that as regards the variation of the elements the disturbing functions are in fact given as explicit functions of the variable elements $\theta, \theta^{\prime}, \phi, \phi^{\prime}$; and where $\phi, \phi^{\prime}$ and therefore also $p, q, p^{\prime}, q^{\prime}$ are only of the order of the disturbing forces.

I proceed to work out this idea, for the present considering the development of the Disturbing Function only as far as the first powers of $p$, $q$, \&c. For comparison with the ordinary theory, observe that in this theory the disturbing function contains only the second powers of the $p, q$, \&c., made use of therein; these are in fact of a form such as $P+p, Q+q, \ldots$ where $P, Q$ are absolute constants and $p, q, \ldots$ are the $p, q, \ldots$ of the present theory; the ordinary theory gives therefore in the disturbing function a series of terms involving $(P+p)^{2},(P+p)(Q+q), \ldots$ which I now take account of only as far as the first powers of $p, q, \ldots$ viz., they are in effect reduced to $P^{2}+2 P p, P Q+P q+Q p, \& c$.... The present theory is thus not now developed to the extent of giving the $p, q, \ldots$ of the ordinary theory in the more complete form as the solutions of a system of simultaneous linear differential equations, but only to the extent of obtaining for these $p, q, \ldots$ respectively the terms which are proportional to the time.

I commence with the following subsidiary problem. Consider a spherical triangle $A B C$ (sides $a, b, c$, angles $A, B, C$, as usual), and taking the side $c$ as constant, but the angles $A$ and $B$ as variable, let $\mathrm{i}$ i be required to find the variations of $C, a, b$ in terms of variations $d A, d B$ and the variable elements $C, a, b$ themselves. Although the geometrical proof would be more simple, I give the analytical one, as it may be useful.

We have

$$
\cos C=-\cos A \cos B+\sin A \sin B \cos c,
$$

and thence

$$
\begin{aligned}
-\sin C d C= & (\sin A \cos B+\cos A \sin B \cos c) d A \\
& +(\sin B \cos A+\sin A \cos B \cos c) d B \\
= & \frac{\sin B \sin c}{\tan b} d A+\frac{\sin A \sin c}{\tan a} d B,
\end{aligned}
$$

that is

or finally

$$
-\frac{\sin C}{\sin c} d C=\frac{\sin B \cos b}{\sin b} d A+\frac{\sin A \cos a}{\sin a} d B,
$$

Next

$$
-d C=\cos b d A+\cos a d B .
$$

or, differentiating,

$$
\sin a=\sin c \frac{\sin A}{\sin C}
$$

$$
\cos a d a=\frac{\sin c}{\sin ^{2} C}(\sin C \cos A d A-\cos C \sin A d C)
$$


or, substituting for $d C$ its value,

that is

$$
\begin{aligned}
& =\frac{\sin c}{\sin ^{2} C}\{d A(\sin C \cos A+\cos C \sin A \cos b)+d B \cos C \sin A \cos a\} \\
& =\frac{\sin c}{\sin ^{2} C}\left\{d A \frac{\sin A \sin b \cos a}{\sin a}+d B \cos C \sin A \cos a\right\}
\end{aligned}
$$

$$
d a=\frac{1}{\sin C}\left\{d A \frac{\sin A}{\sin a} \sin b+d B \cos C \sin A\right\} \div \frac{\sin C}{\sin c}
$$

or, on the right-hand writing $\frac{\sin A}{\sin a}$ instead of $\frac{\sin C}{\sin c}$, this is

and similarly

$$
d a=\frac{1}{\sin C}(d A \sin b+d B \cos C \sin a)
$$

$$
d b=\frac{1}{\sin C}(d B \sin a+d A \cos C \sin b)
$$

Now let the continuous lines represent the orbits of $m, m^{\prime}$ at certain dates, $O, Q$ the origins of longitude therein; and the dotted lines the variable orbits of the planets respectively.

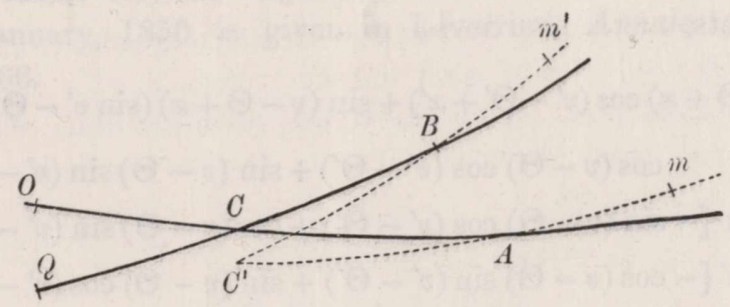

Write

$$
\begin{aligned}
& O C=\Theta, \quad C A=\theta, \quad \angle C A C^{\prime}=\phi \\
& Q C=\Theta^{\prime}, \quad C B=\theta^{\prime}, \quad \angle C B C=\phi^{\prime}, \\
& \angle C=\Phi .
\end{aligned}
$$

Then, answering to the notation of the lemma, we have

whence

$$
\begin{aligned}
& a=\theta^{\prime}, \quad b=\theta, \quad C=\Phi, \quad d A=\phi, \quad d B=-\phi^{\prime}, \\
& \text { or say }=\tan \phi, \quad=-\tan \phi^{\prime} \text {, }
\end{aligned}
$$

$$
\begin{aligned}
C^{\prime} B & =a+d a \\
& =\theta^{\prime}+\frac{1}{\sin \Phi}\left(\tan \phi \sin \theta-\tan \phi^{\prime} \cos \Phi \sin \theta^{\prime}\right) \\
& =\theta^{\prime}+\frac{1}{\sin \Phi}\left(p-p^{\prime} \cos \Phi\right)
\end{aligned}
$$




$$
\begin{aligned}
C^{\prime} A & =b+d b \\
& =\theta+\frac{1}{\sin \Phi}\left(-\tan \theta^{\prime} \sin \phi^{\prime}+\tan \phi \cos \Phi \sin \theta\right), \\
& =\theta-\frac{1}{\sin \Phi}\left(p^{\prime}-p \cos \Phi\right), \\
\angle C^{\prime} & =C+d C=\Phi-\cos \theta \tan \phi+\cos \theta^{\prime} \tan \phi^{\prime},=\Phi-q+q^{\prime} .
\end{aligned}
$$

Suppose $v, v^{\prime}$ are the longitudes of the planets in their two orbits respectively; that is

$$
\begin{aligned}
& v=O A+A m=\Theta+\theta+A m, \\
& v^{\prime}=Q B+B m^{\prime}=\Theta+\theta^{\prime}+B m^{\prime},
\end{aligned}
$$

whence

$$
\begin{aligned}
C^{\prime} m & =C^{\prime} A+A m,=v-\Theta-\frac{1}{\sin \Phi}\left(p^{\prime}-p \cos \Phi\right) \\
C^{\prime} m^{\prime} & =C^{\prime} B+B m,=v^{\prime}-\Theta^{\prime}+\frac{1}{\sin \Phi}\left(p-p^{\prime} \cos \Phi\right), \\
\angle C^{\prime}= & \Phi-q+q^{\prime}
\end{aligned}
$$

say these values are $v-\Theta+x, v^{\prime}-\Theta^{\prime}+x^{\prime}, \Phi+y$. Then if $H$ is the angular distance $\mathrm{mm}^{\prime}$ of the two planets,

$$
\begin{aligned}
& \cos \bar{H}=\cos (v-\Theta+x) \cos \left(v^{\prime}-\Theta^{\prime}+x^{\prime}\right)+\sin (v-\Theta+x)\left(\sin v^{\prime}-\Theta^{\prime}+x^{\prime}\right) \cos (\Phi+y), \\
& =\quad \cos (v-\Theta) \cos \left(v^{\prime}-\Theta^{\prime}\right)+\sin (v-\Theta) \sin \left(v^{\prime}-\Theta^{\prime}\right) \cos \Phi \\
& +x\left[-\sin (v-\Theta) \cos \left(v^{\prime}-\Theta^{\prime}\right)+\cos (v-\Theta) \sin \left(v^{\prime}-\Theta^{\prime}\right) \cos \Phi\right] \\
& +x^{\prime}\left[-\cos (v-\Theta) \sin \left(v^{\prime}-\Theta^{\prime}\right)+\sin (v-\Theta) \cos \left(v^{\prime}-\Theta^{\prime}\right) \cos \Phi\right] \\
& +y\left[-\sin (v-\Theta) \sin \left(v^{\prime}-\Theta^{\prime}\right) \sin \Phi\right] \text {, } \\
& =\cos H+\nabla \text { suppose. }
\end{aligned}
$$

The disturbing function for the planet $m$ disturbed by $m^{\prime}$ is

$$
\Omega=m^{\prime}\left\{\frac{1}{\sqrt{r^{2}+r^{\prime 2}-2 r r^{\prime} \cos \bar{H}}}-\frac{r \cos H}{r^{\prime 2}}\right\},
$$

( $\Omega=-R$, if $R$ is the disturbing function of the Mécanique Céleste); and the term hereof which involves $\nabla$ is

$$
=\nabla \frac{d \Omega}{d \cdot \cos H}
$$

where after the differentiation $\cos H$ is replaced by $\cos H$,

$$
=m^{\prime}\left\{\frac{r r^{\prime}}{\left(r^{2}+r^{\prime 2}-2 r r^{\prime} \cos H\right)^{\frac{3}{2}}}+\frac{r}{r^{\prime 2}}\right\} \nabla,
$$


viz., this is a linear function of $x, x^{\prime}, y$, that is of $p, q, p^{\prime}, q^{\prime}$, with coefficients which of course involve the other variable elements and the time; but it will be remembered that $\Theta, \Theta^{\prime}, \Phi$ are not variable elements, but are absolute constants. The variations of $p$ depend upon $\frac{d \Omega}{d q}$ and those of $q$ on $\frac{d \Omega}{d p}$, and the quantities $p, q, p^{\prime}, q^{\prime}, \ldots$ disappear from these differential coefficients $\frac{d \Omega}{d q}, \frac{d \Omega}{d p}$; that is, disregarding periodic terms, and the variations of the elements, we $\cdot$ obtain $\frac{d p}{d t}, \frac{d q}{d t}$ as absolute constants, or reckoning the time from the epoch belonging to the fixed orbit of $m$, we have $p, q$ as mere multiples of the time ( $p=A t, q=B t$, where $A$ and $B$ are constants); agreeing with the statement preceding the investigation.

Observe that the $p, q$, as used above, have reference not only to the fixed orbit of $m$, but also to the node thereon of the fixed orbit of $m^{\prime}$ : we may, if we please, write $\mathrm{p}=\tan \phi \sin (\Theta+\theta), \mathrm{q}=\tan \phi \cos (\Theta+\theta)$, that is, $\mathrm{p}=q \sin \Theta+p \cos \Theta, Q=q \cos \Theta-p \sin \Theta$ (or $p=p \cos \Theta-q \sin \Theta, q=P \sin \Theta+q \cos \Theta$ ), and in place of $p, q$ introduce into the formulæ $\mathrm{p}$ and $\mathrm{q}$, which have reference only to the fixed orbit of $m$, and similarly writing $\mathrm{p}^{\prime}-\tan \phi^{\prime} \sin \left(\Theta^{\prime}+\theta^{\prime}\right), \quad \mathrm{q}^{\prime}=\tan \phi^{\prime} \cos \left(\Theta+\theta^{\prime}\right)$, instead of $p^{\prime}, q^{\prime}$ introduce $p^{\prime}, q^{\prime}$ which have reference only to the fixed orbit of $m^{\prime}$.

I remark that a table for the relative positions of the orbits of the eight Planets for the Epoch 1st January, 1850, is given in Leverrier's Annales de l'Observ. de Paris, t. II. $(1856)$, pp. $64-66$. 